\title{
Eficacia de la
}

Telepsicología en intervenciones del área clínica y de la

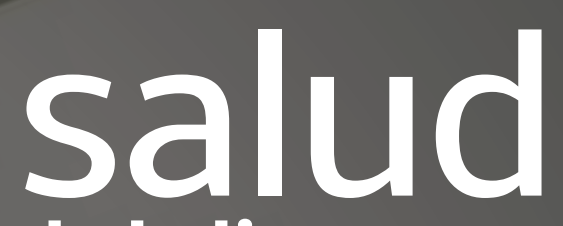

\section{una revisión sistemática de la literatura}

fficacy of telepsychology in clinical and health interventions: a systematic

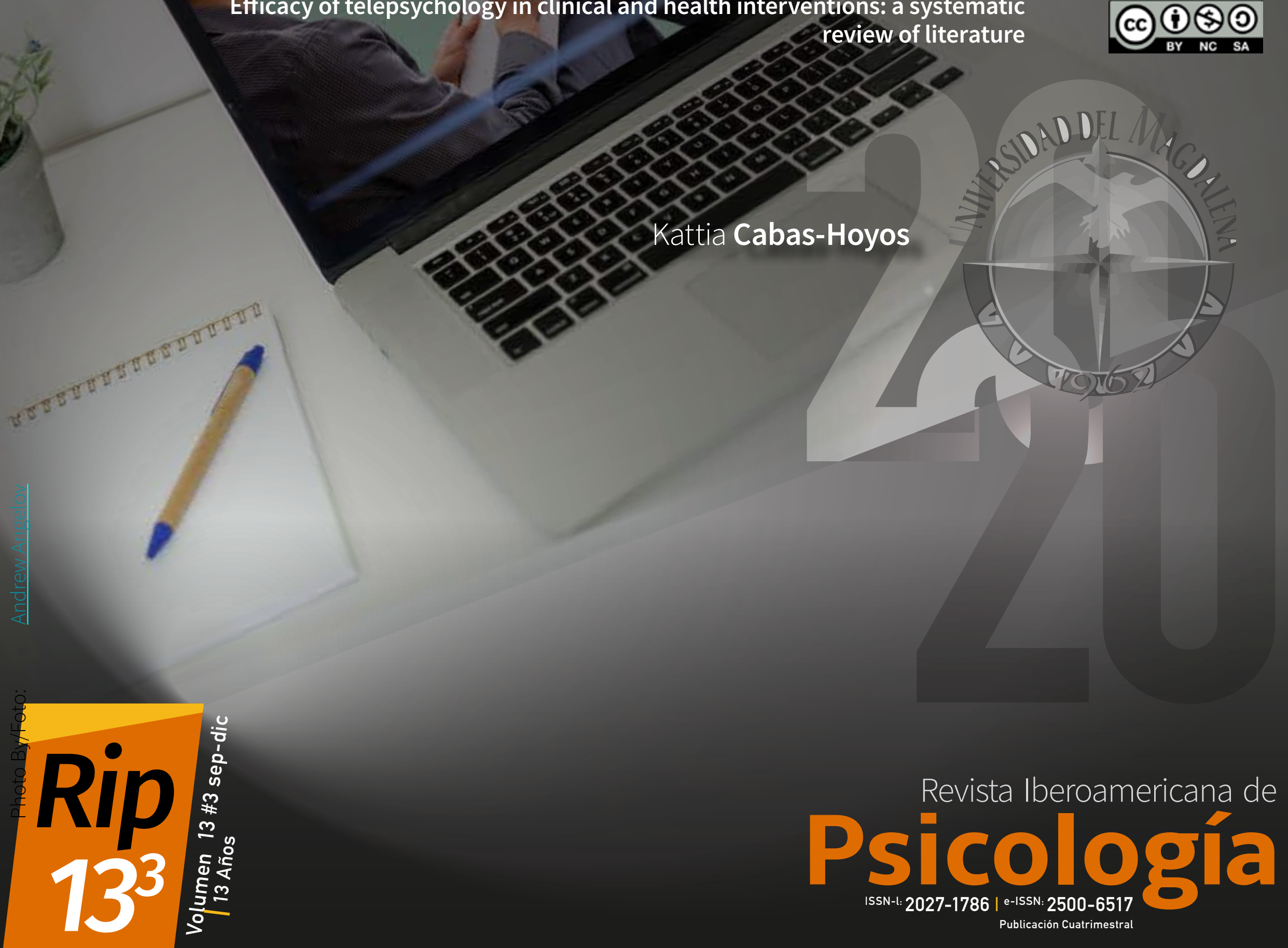


ID:

\section{7-1786.rip.13306}

Title: Efficacy of telepsychology in clinical and health interventions

Subtitle: A systematic review of literature

Título: Eficacia de la Telepsicología en intervenciones del área clínica y de la salud

Subtítulo: Una revisión sistemática de la literatura

Alt Title / Título alternativo:

[en]:

Efficacy of telepsychology in clinical and health interventions: a systematic review of literature

[es]:

Eficacia de la Telepsicología en intervenciones del área clínica y de la salud: una revisión sistemática de la literatura

Author (s) / Autor (es):

Cabas-Hoyos

Keywords / Palabras Clave:

[en]: Telepsychology; videoconference; internet-based intervention; telephone-based intervention

[es]: Telepsicología; videoconferencias; intervención vía internet; intervención vía telefónica

Submited: 2020-07-06

Acepted: 2020-09-21

\section{Resumen}

La telepsicología es una herramienta de intervención prometedora que puede aumentar el acceso a los servicios psicológicos reduciendo las barreras de accesibilidad al tratamiento. De este modo, se realizó una búsqueda sistemática en bases de datos con el objetivo de revisar la evidencia sobre la eficacia en intervenciones de telepsicología en el área clínica y de la salud en los últimos 10 años. De un total de 86 registros identificados, 15 estudios empíricos cumplieron con los criterios de selección y fueron incluidos en la revisión, de los cuales,: 6 investigaron la efectividad de la telepsicología mediante videoconferencia; 3 mediante intervención telefónica, y 6 estudios por la modalidad vía internet

\section{Abstract}

Telepsychology is a promising intervention tool that can increase access to psychological services by reducing accessibility barriers

to treatment. Thus, a systematic search was carried out in databases with the aim of reviewing the evidence on the efficacy of telepsychology interventions in the clinical and health area in the last 10 years. From a total of 86 records identified, 15 empirical studies met the selection criteria and were included in the review, of which, 6 investigated the effectiveness of telepsychology through videoconferencing; 3 by telephone intervention, and 6 studies by the online modality

\section{Citar como:}

Cabas-Hoyos, K. . (2020). Eficacia de la Telepsicología en intervenciones del área clínica y de la salud: Una revisión sistemática de la literatura. Revista Iberoamericana de Psicología, 13 (3), [pgIn]-[pgOut]. Obtenido de: https://reviberopsicologia.ibero.edu.co/article/view/1891

Dra Kattia Cabas-Hoyos, Ma Psi

AutorlD: $\quad$ https://scholar.google.com/citations? user=Rl19YvcAAAAJ\&hl=es

Research ID: $\quad$ https://www.scopus.com/authid/detail.uri?authorld=36089034100 ORCID: $\quad$ https://orcid.org/0000-0002-1548-9430

Source I Filiacion:

Universidad del Magdalena

BIO:

Doctora, Psicóloga clínica, Docente Universitaria e Investigadora Asociada (MinCiencias), con intereses investigativos tanto en Telepsicología como el desarrollo y uso de nuevas tecnologías aplicadas a la salud mental

City I Ciudad:

Santa Marta [co]

e-mail:

kcabas@unimagdalena.edu.co 


\section{Eficacia de la Telepsicología en intervenciones del área clínica y de la salud una revisión sistemática de la literatura}

Efficacy of telepsychology in clinical and health interventions: a systematic review of literature

Kattia Cabas-Hoyos

La telepsicología, es decir, la prestación de servicios psicológicos a través de la tecnología es un campo amplio y abarca diversas modalidades a través formatos de intervención como teléfono, correo electrónico, videoconferencia (VC), aplicaciones móviles o programas basados en internet (Nelson, Bui \& Velásquez, 2011; American Psychological Associations [APA], 2013). Estos formatos pueden ser autodirigidos o en ocasiones contar con la interacción en tiempo real de un especialista, es decir, la intervención puede ser "sincrónica" o "asincrónica” (Wilson \& Maeder, 2015; Varker, Brand, Ward, Terhaag \& Phelps, 2018).

Las intervenciones sincrónicas se caracterizan por interacciones visuales o auditivas entre emisores y receptores de forma simultánea, por lo tanto, es el formato más similar al tratamiento tradicional en persona o cara a cara. Un ejemplo de este tipo de intervención serían los tratamientos telefónicos, VC o aquellas interacciones por texto. Las intervenciones asincrónicas, por su parte, implican interacciones no simultáneas que se caracterizan por realizarse mediante correo electrónico, siendo esta la herramienta más común (Osenbach, O’Brien, Mishkind \& Smolenski, 2013; Drum \& Littleton, 2014).

La oferta de servicios de telepsicología, sin embargo, ha provocado gran debate en la comunidad científica y profesional debido a las dificultades que podrían presentarse en el establecimiento de la relación terapéutica, el manejo de crisis (Varker et al., 2018; Sucala et al., 2012) y las dudas sobre la efectividad de las intervenciones (Hilty et al., 2015; Backhaus, et al., 2012). Igualmente, en la intervención asincrónica como el correo electrónico o chat, se contempla como dificultad la imposibilidad de observar la comunicación no verbal o cualidades de la voz, problemas de seguridad y privacidad sobre el proceso del servicio prestado (Clinton, Silverman \& Brendel, 2010; DiLillo \& Gale, 2011). 


\section{Eficacia de la Telepsicología en intervenciones del área clínica y de la salud}

Una revisión sistemática de la literatura

Lo anterior, además, ha significado un desafío para aquellos países que han enfrentado a lo largo de los años desafíos geográficos en la prestación de servicios en el área clínica y de la salud (Frueh, 2015; Mehrotra et al., 2016), esto debido a que entre mayores son las distancias geográficas, menor es el uso de servicios psicológicos, existiendo una restricción mayor en la disponibilidad de profesionales especializados en el tratamiento de trastornos mentales (Handley et al., 2014; Meadows, Enticott, Inder, Russell \& Gurr, 2015). Sin embargo, esta no es la única barrera que ha existido a lo largo de los años para buscar o recibir tratamiento de salud mental en áreas remotas, también se han presentado preocupaciones sobre el estigma, la edad, dificultad para transportarse, preocupaciones sobre el costo de tratamiento o residir en zonas rurales.

A partir de lo anterior, la evidencia sobre la efectividad de la telepsicología ha aumentado en la última década. Las revisiones sistemáticas que han combinado ambas modalidades de intervención, es decir, sincrónica y asincrónica, han indicado que la telepsicología es generalmente tan efectiva como la modalidad cara a cara (Tudor-Tulbure, 2011; Backhaus et al., 2012; Varker et al., 2018). La literatura demuestra, además, que mediante el uso de la tecnología es posible proporcionar intervenciones y programas de tratamiento completos, con observancia de los criterios éticos, y que resulta especialmente útil en aquellas situaciones en las que el acceso a servicios de manera oportuna está limitado debido a la ubicación geográfica, las condiciones de salud, o temores específicos asociados con el contacto personal posibilitando además un tratamiento desde la privacidad de la casa (Frueh, 2015; Riding-Malon \& Werth Jr, 2014; Soto-Pérez \& FrancoMartín, 2018; Acero et al., 2020).

Por consiguiente, el objetivo de esta revisión es determinar la eficacia de la telepsicología mediante videoconferencia, teléfono e internet en el área clínica y de la salud para el tratamiento de trastornos mentales en los últimos diez años, comparando si las intervenciones sincrónicas y asincrónicas son tan eficaces como los tratamientos cara a cara y actualizando así la literatura existente tomando sólo ese rango de tiempo.

\section{Métodos}

El presente estudio se caracteriza por ser una revisión sistemática de la literatura (SLR), siendo un procedimiento que recopila, evalúa de forma crítica, analiza y presenta los resultados de estudios o trabajos de investigación a través de un proceso sistemático que responde a una pregunta problema o tema de interés. Es "sistemática" debido a que adopta una metodología consistente y ampliamente aceptada (Ferreras-Fernández, Martín-Rodero, García-Peñalvo, \& Merlo-Vega, 2016; Pati \& Lorusso, 2017).

\section{Búsqueda e identificación de}

\section{estudios:}

Se realizó una búsqueda en las bases de datos PubMed, ScienceDirect, Scientific Electronic Library Online (SciELO) y Cambridge Core. Fue utilizada la siguiente combinación de palabras: "efficacy" OR "effectiveness" AND "telepsychology" AND "Cognitive Behavioral Therapy" OR "CBT"; "efficacy" OR "effectiveness" AND "telepsychology" AND "psychotherapy" OR "therapy"; "efficacy" OR "effectiveness" AND "telepsychology" AND "intervention"; "efficacy" OR "effectiveness" AND "telephone-based intervention" OR "telephone-based therapy"; "effi- cacy" OR "effectiveness" AND "videoconference-based intervention" OR "videoconference-based therapy"; "efficacy" OR "effectiveness" AND "internet-based intervention" OR "internet-based therapy", y sus correlatos en portugués y español.

\section{Criterios de selección:}

Fueron incluidos en la revisión artículos originales en inglés, portugués o español publicados entre el 2010 y el 2020 con muestras caracterizadas por niños, adolescentes y/o adultos. Así mismo, se incluyeron aquellos trabajos que implementaron la telepsicología en intervenciones clínicas y de la salud a través de VC, internet o mediante recursos telefónicos. Esto último quiere decir que se tomaron en cuenta intervenciones sincrónicas y asincrónicas. Fueron excluidas revisiones de literatura, validación de instrumentos, disertaciones y tesis. Igualmente, aquellos artículos publicados antes del 2010 y los que usaban intervenciones cara a cara no fueron tomados en cuenta, salvo aquellos que compararon la efectividad de dichos métodos de intervención. Tampoco fueron seleccionadas las propuestas de investigación.

\section{Evaluación de la calidad o valoración crítica:}

Con el fin de reducir los posibles sesgos y aumentar la calidad metodológica de esta revisión y de los artículos que hacen parte de la muestra final, fue usado el checklist propuesto por Downs \& Black (1998). El instrumento contiene 26 ítems con respuestas dicotómicas 1: "sí"; 0: "no". El cuestionario propone como media M=14.0 y como desviación típica D.T=6.39 para estudios controlados y aleatorizados; para aquellos no aleatorizados la media es $M=11,7$ y la desviación típica es D.T=4.64. Las preguntas 1, 2, 3, 4, 6, 10, 11, 13, 17, 18 y 20 obtuvieron respuestas positivas para la evaluación de los estudios seleccionados, y la sumatoria de todas dio como resultado un total de 11 puntos, esto refleja buena calidad metodológica de las investigaciones seleccionadas para la revisión.

La calidad metodológica de los estudios es sustentada porque las hipótesis u objetivos de los estudios fueron claramente descritos; el objetivo de cada estudio se describió en la introducción y en la metodología; y las características de los participantes fueron descritas de forma clara. Así mismo, los tipos de intervención en pacientes con TEPT y controles también se describen de manera detallada y los resultados se exponen basados en significancia estadística. De la misma forma, los sujetos reclutados fueron invitados a participar y los estudios con controles, lista de espera o aquellos que tuvieron seguimiento fueron claramente descritos.

\section{Plan de análisis de datos:}

Para lograr una sistematización clara y objetiva de los resultados, se siguieron las directrices de la declaración Preferred Reporting Items for Systematic Reviews and Meta-Analyses (PRISMA), determinando aquellos estudios elegibles que conformarían la muestra final mediante la identificación, revisión, selección y análisis de los mismos disminuyendo posibles sesgos (Moher, Liberati, Tetzlaff, Altman \& The Prima Group, 2009; Manterola, Astudillo, Arias \& Claros, 2013).

En el proceso de búsqueda explicado en la Figura 1, se aprecia que 15 artículos originales cumplieron con los criterios de inclusión y conformaron la muestra final. Los datos de cada investigación fueron clasificados en el siguiente orden: estudio, N (fem/masc), edad M (D.T), 
diagnóstico, diseño, país, objetivo, resultados, limitaciones. Las tablas estuvieron distribuidas según el tipo de intervención: videoconferencia (ver Tabla 1), telefónica (ver Tabla 2) e internet (ver Tabla 3).

\section{Resultados}

En la búsqueda y selección de los artículos que harían parte de la muestra final, se identificaron inicialmente 86 estudios a través de la búsqueda en las bases de datos mencionadas anteriormente incluyendo 2 registros adicionales a través de otras fuentes. Al remover 20 artículos duplicados, se revisaron 67 registros para posteriormente excluir 27 por no cumplir con los criterios de selección, de esta manera, 40 textos completos fueron evaluados para elegibilidad, de los cuales se excluyeron 25, pues: a) 8 eran artículos de revisión; b) 3 fueron disertaciones o tesis; c) 14 no eran intervenciones a través de telepsicología. Finalmente, 15 estudios cumplieron con los criterios de selección y conformaron la muestra de la presente revisión.

Figura 1 Flujograma PRISMA de búsqueda y selección de documentos para la revisión.
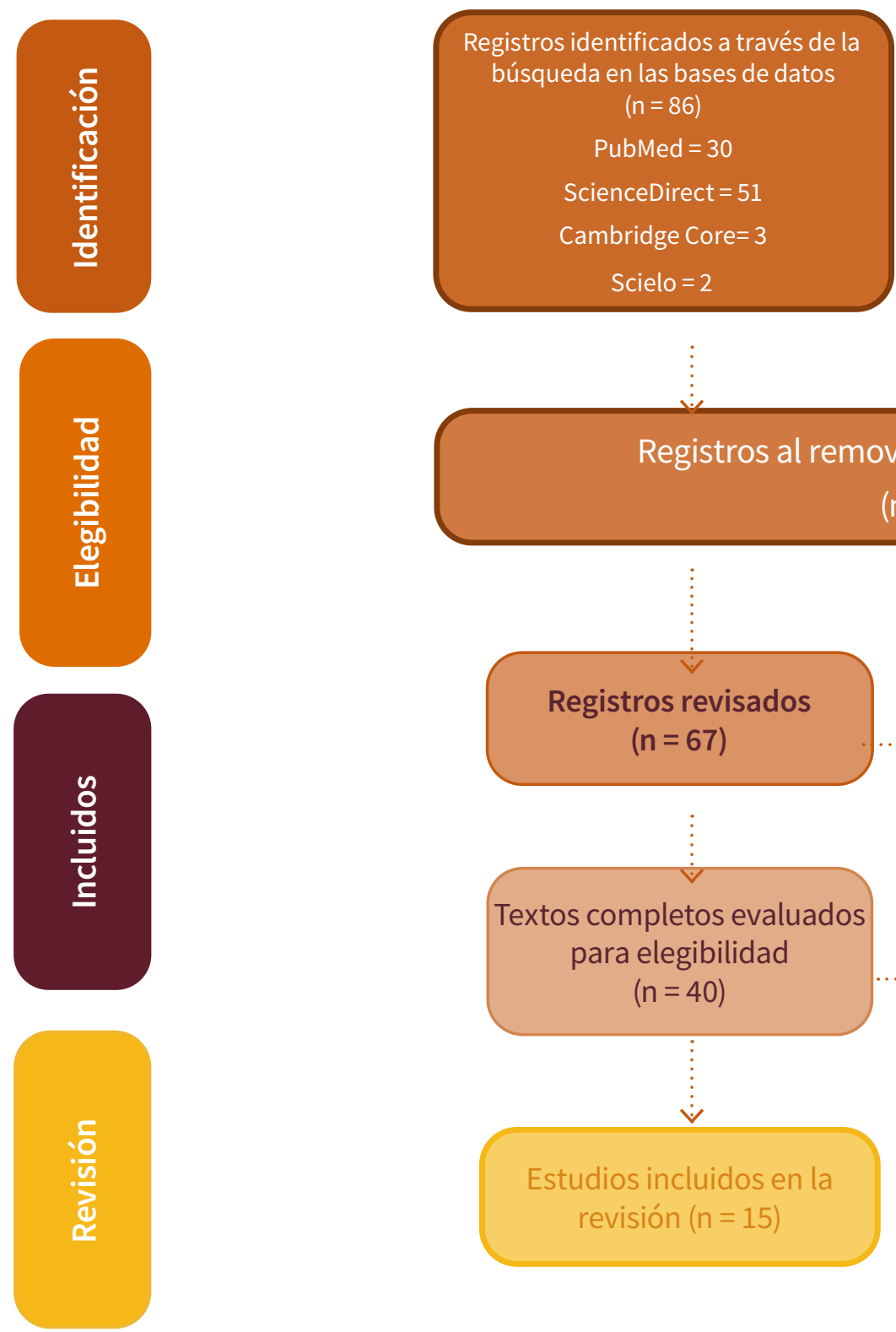

Tabla 1 Intervenciones mediante videoconferencia

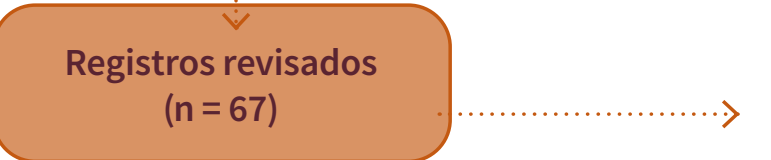

Textos completos evaluado para elegibilidad $(n=40)$

¿

Estudios incluidos en la revisión $(n=15)$
Registros excluidos $(n=27)$

No cumplen con los criterios de inclusión

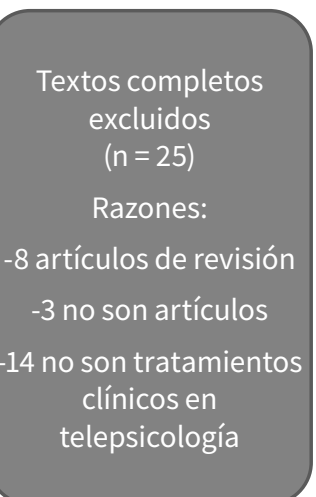

\begin{tabular}{|c|c|c|c|c|c|c|c|c|}
\hline Estudio & $\begin{array}{c}\mathrm{N} \\
\text { (fem/ } \\
\text { masc) }\end{array}$ & $\begin{array}{l}\text { Edad } \\
\text { M (D.T) }\end{array}$ & Diagnóstico & Diseño & País & Objetivo & Resultados & Limitaciones \\
\hline $\begin{array}{l}\text { Escudero et } \\
\text { al. (2017) }\end{array}$ & $\begin{array}{c}1 \\
(0 / 1)\end{array}$ & 52 años & $\begin{array}{l}\text { TEPT, trastorno } \\
\text { de depresión } \\
\text { mayor, fobia } \\
\text { social y TOC. }\end{array}$ & $\begin{array}{l}\text { Pre-Post } \\
\text { seguimiento }\end{array}$ & España & $\begin{array}{l}\text { Evaluar la } \\
\text { efectividad } \\
\text { clínica de } \\
\text { la terapia } \\
\text { cognitivo- } \\
\text { conductual } \\
\text { centrada en el } \\
\text { trauma (TCC- } \\
\text { CT) mediante } \\
\text { VC. }\end{array}$ & $\begin{array}{c}\text { Tras } 36 \text { sesiones } \\
\text { de tratamiento, se } \\
\text { produjo una reducción } \\
\text { clínicamente significativa } \\
\text { de la sintomatología } \\
\text { postraumática, ansiosa } \\
\text { y depresiva, la cual se } \\
\text { mantuvo estable a largo } \\
\text { plazo. }\end{array}$ & $\begin{array}{l}\text { El diseño mismo } \\
\text { del estudio, a saber, } \\
\text { estudio de caso. }\end{array}$ \\
\hline
\end{tabular}


Eficacia de la Telepsicología en intervenciones del área clínica y de la salud

Una revisión sistemática de la literatura

\begin{tabular}{|c|c|c|c|c|c|c|c|c|}
\hline Estudio & $\begin{array}{c}\mathrm{N} \\
(\mathrm{fem} / \\
\text { masc) }\end{array}$ & $\begin{array}{c}\text { Edad } \\
\text { M (D.T) }\end{array}$ & Diagnóstico & Diseño & País & Objetivo & Resultados & Limitaciones \\
\hline $\begin{array}{l}\text { Germain, } \\
\text { vdsMarchand, } \\
\text { Bouchard, } \\
\text { Guay \& Drouin } \\
\text { (2010) }\end{array}$ & $\begin{array}{c}46 \\
\text { Grupo } \\
\text { control } \\
\text { VC: } \\
(11 / 6) \\
\\
\text { Grupo } \\
\text { Cara a } \\
\text { cara: } \\
\text { (16/13) }\end{array}$ & $\begin{array}{l}\text { Rango: } \\
\text { 18-65 } \\
\text { años }\end{array}$ & $\begin{array}{c}\text { Trastorno } \\
\text { por Estrés } \\
\text { Postraumático } \\
\text { (TEPT) }\end{array}$ & $\begin{array}{c}\text { Ensayo controlado } \\
\text { aleatorizado: VC vs. } \\
\text { Cara a cara }\end{array}$ & Canadá & $\begin{array}{c}\text { Evaluar } \\
\text { la alianza } \\
\text { terapéutica } \\
\text { en individuos } \\
\text { TEPT que } \\
\text { fueron tratados } \\
\text { mediante } \\
\text { terapia de VC o } \\
\text { una terapia cara } \\
\text { a cara. }\end{array}$ & $\begin{array}{l}\text { Los resultados indican } \\
\text { que una alianza } \\
\text { terapéutica puede } \\
\text { desarrollarse muy bien } \\
\text { en ambas condiciones } \\
\text { de tratamiento y que } \\
\text { no hay una diferencia } \\
\text { significativa entre los dos. }\end{array}$ & $\begin{array}{c}\text { Se produjeron } \\
\text { problemas técnicos } \\
\text { con las sesiones de } \\
\text { VC. }\end{array}$ \\
\hline
\end{tabular}

Tabla 1 (continuación)

\begin{tabular}{|c|c|c|c|c|c|c|c|c|}
\hline Estudio & $\begin{array}{c}\mathrm{N} \\
\text { (fem/masc) }\end{array}$ & $\begin{array}{c}\text { Edad } \\
\text { M (D.T) }\end{array}$ & Diagnóstico & Diseño & País & Objetivo & Resultados & Limitaciones \\
\hline
\end{tabular}

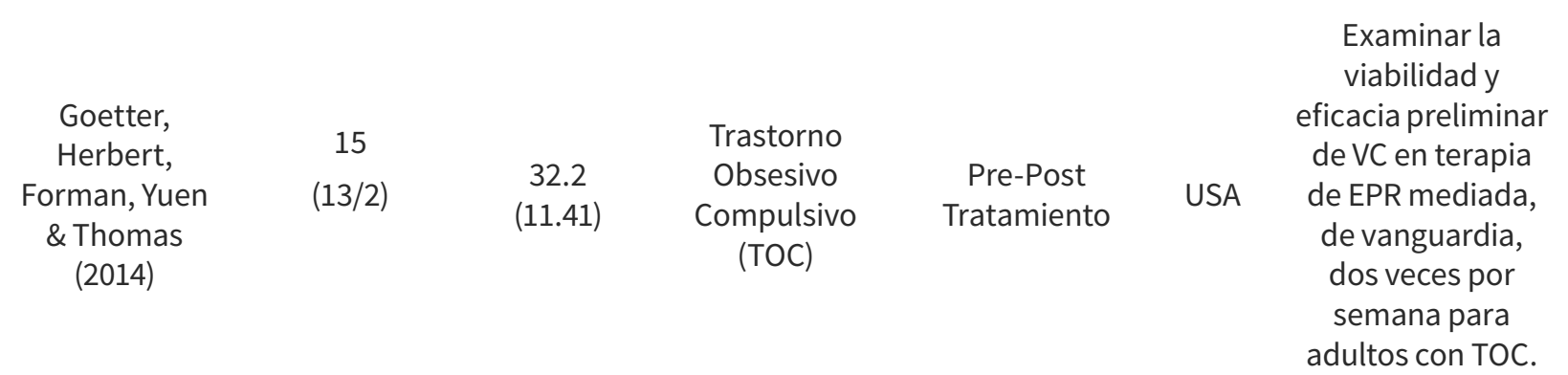

La EPR mediante CV logró mejoras significativas en los síntomas de TOC del grupo. Entre las personas que completaron el seguimiento de 3 meses, el $30 \%$ de los participantes ya no cumplieron con los criterios DSM-IVTR para

Pequeño tamaño de la muestra. La integridad del tratamiento y la confiabilidad entre evaluadores no se tuvo en cuenta.

$$
\text { el TOC. }
$$

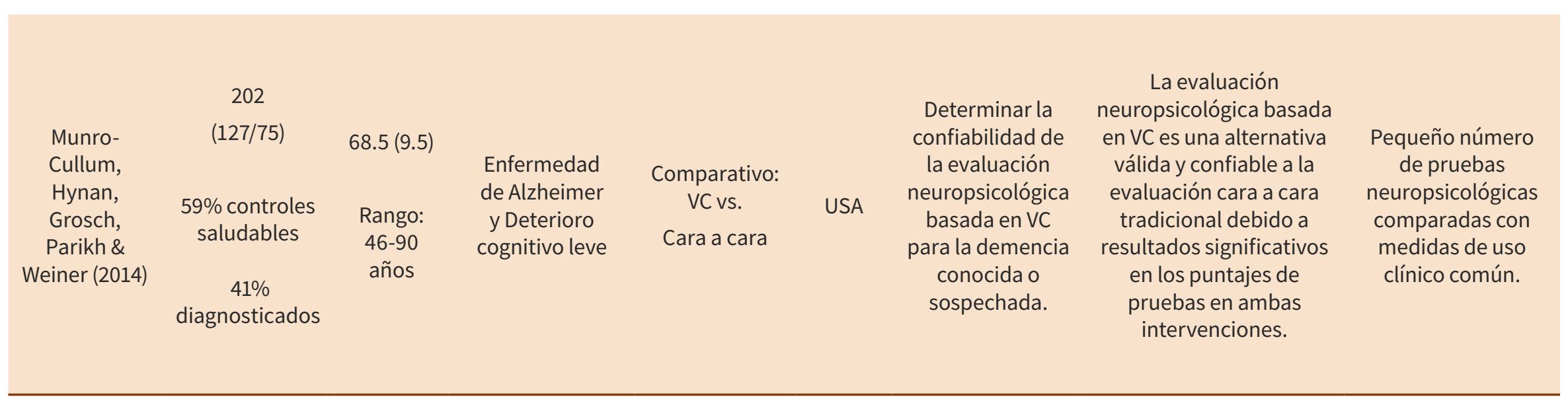

Tabla 1 (continuación)

$\begin{array}{ccc}\text { Estudio } & \begin{array}{c}\text { Nzzz(fem/ } \\ \text { masc) }\end{array} & \text { Edad } \\ & \text { M(D.T) }\end{array} \quad$ Diagnóstico Diseño $\quad$ País $\quad$ Objetivo $\quad$ Resultados $\quad$ Limitaciones

26

Stubbings, $\quad \begin{gathered}\text { Grupo VC: } \quad 30(11) \\ (8 / 6)\end{gathered}$

Rees,

Roberts

\& Kane

(2013)

$(8 / 6)$

$30(11)$

Trastornos

Ensayo

controlado

aleatorizado: Australia

Rango: 18-

del estado

de ánimo y

ansiedad

vC vs.

Cara a cara

Cara a

cara:

(7/5)

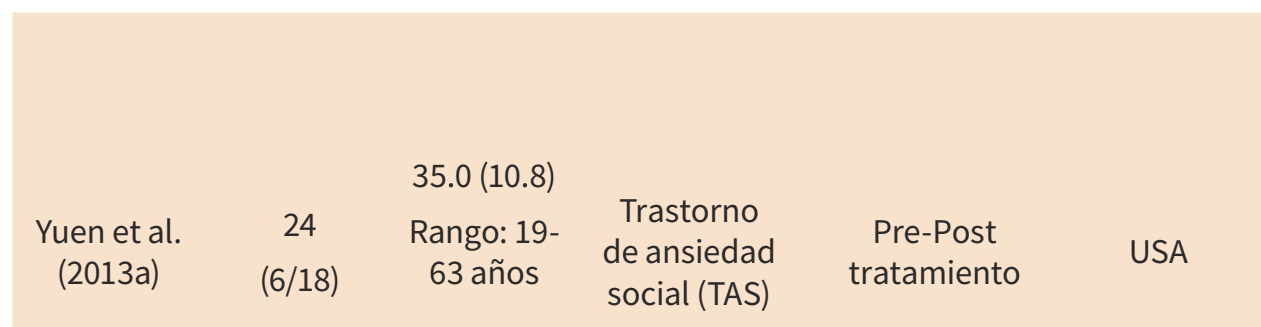

Comparar la efectividad de la TCC administrada por VC con la terapia en persona para una cohorte de diagnóstico mixta.
La TCC fue efectiva para reducir los síntomas de depresión, ansiedad y estrés, y para aumentar la calidad de vida tanto en condiciones de persona como en VC.
Se incluyó sólo un terapeuta en el estudio. El tamaño limitado de la muestra debilita la generalización de los hallazgos.
Evaluar la viabilidad, aceptabilidad y eficacia de una intervención conductual mediante VC para tratar a adultos TAS.
Los análisis revelaron un pretratamiento significativo para las mejoras de seguimiento en la ansiedad social con resultados comparables o mayores a aquellos publicados en estudios que ofrecen TCC en persona TAS.
Los ejercicios de exposición en sesión se limitaron a escenarios que podrían completarse mediante VC. 


\begin{tabular}{|c|c|c|c|c|c|c|c|c|}
\hline Estudio & $\begin{array}{c}\mathrm{N} \\
\text { (fem/ } \\
\text { masc) }\end{array}$ & $\begin{array}{c}\text { Edad } \\
\text { M (D.T) }\end{array}$ & Diagnóstico & Diseño & País & Objetivo & Resultados & Limitaciones \\
\hline $\begin{array}{l}\text { Ben-Seev et } \\
\text { al. (2014) }\end{array}$ & $\begin{array}{c}32 \\
(12 / 20)\end{array}$ & $45.9(8.78)$ & Esquizofrenia & Pre-Post tratamiento & USA & $\begin{array}{c}\text { Evaluar la } \\
\text { viabilidad, } \\
\text { aceptabilidad y } \\
\text { eficacia preliminar } \\
\text { de una intervención } \\
\text { de teléfono } \\
\text { inteligente para la } \\
\text { esquizofrenia. }\end{array}$ & $\begin{array}{l}\text { Las pruebas t de } \\
\text { muestras pareadas } \\
\text { encontraron } \\
\text { reducciones } \\
\text { significativas en los } \\
\text { síntomas psicóticos, } \\
\text { la depresión y la } \\
\text { psicopatología } \\
\text { general, después } \\
\text { de } 1 \text { mes de uso de } \\
\text { FOCUS. }\end{array}$ & $\begin{array}{c}\text { Ausencia de grupo } \\
\text { control, por lo que no } \\
\text { es posible determinar } \\
\text { si las mejoras clínicas } \\
\text { estaban relacionadas } \\
\text { con FOCUS. }\end{array}$ \\
\hline $\begin{array}{l}\text { Himelhoch } \\
\text { et al. (2013) }\end{array}$ & $\begin{array}{c}\text { Grupo } \\
\text { TCC por } \\
\text { teléfono } \\
(12 / 4) \\
\\
\text { Grupo } \\
\text { Cara a } \\
\text { cara } \\
(13 / 5)\end{array}$ & $\begin{array}{l}46.78 \\
(8.87)\end{array}$ & $\begin{array}{c}\text { Trastorno de } \\
\text { depresión } \\
\text { mayor }\end{array}$ & $\begin{array}{c}\text { Ensayo controlado } \\
\text { aleatorizadoTeléfono } \\
\text { vs. } \\
\text { Cara a cara }\end{array}$ & USA & $\begin{array}{l}\text { Evaluar una } \\
\text { TCC mediante } \\
\text { intervención } \\
\text { telefónica en } \\
\text { comparación con la } \\
\text { terapia cara a cara } \\
\text { entre individuos } \\
\text { con depresión } \\
\text { infectados con } \mathrm{VIH} \text {. }\end{array}$ & $\begin{array}{c}\text { En ambas } \\
\text { modalidades } \\
\text { se produjeron } \\
\text { reducciones } \\
\text { significativas en los } \\
\text { síntomas depresivos. } \\
\text { El grupo de TCC } \\
\text { por teléfono fue } \\
\text { significativamente } \\
\text { más propenso } \\
\text { a su adherencia } \\
\text { a la medicación } \\
\text { comparada con cara } \\
\text { a cara. }\end{array}$ & $\begin{array}{l}\text { Recepción adicional } \\
\text { de intervenciones } \\
\text { adicionales de salud } \\
\text { mental por parte de } \\
\text { un gran número de } \\
\text { participantes. }\end{array}$ \\
\hline
\end{tabular}

Tabla 2 (continuación)

\begin{tabular}{|c|c|c|c|c|c|c|c|c|}
\hline Estudio & $\begin{array}{c}\mathrm{N} \\
\text { (fem/ } \\
\text { masc) }\end{array}$ & $\begin{array}{c}\text { Edad } \\
\text { M (D.T) }\end{array}$ & Diagnóstico & Diseño & País & Objetivo & Resultados & Limitaciones \\
\hline
\end{tabular}

\begin{tabular}{|c|c|c|c|}
\hline \\
\hline \multicolumn{4}{|c|}{ rupo Cara } \\
\hline $\begin{array}{l}\text { a cara } \\
(127 / 35)\end{array}$ & $475(13,5)$ & Trastorno de & $\begin{array}{c}\text { Ensayo } \\
\text { controlado }\end{array}$ \\
\hline $\begin{array}{l}\text { Grupo } \\
\text { TCC por }\end{array}$ & $4.0(13.0)$ & $\begin{array}{l}\text { depresión } \\
\text { mayor }\end{array}$ & $\begin{array}{l}\text { aleatorizado: } \\
\text { Teléfono vs. }\end{array}$ \\
\hline $\begin{array}{l}\text { teléfono } \\
(125 / 38)\end{array}$ & 47.8 (12.6) & & Cara a cara \\
\hline
\end{tabular}

Examinar si la terapia cognitiva conductual administrada por teléfono (T-TCC) reduce la deserción y no es inferior a la TCC presencial en el tratamiento de la depresión.
Entre los pacientes de atención primaria con depresión, proporcionar TCC por teléfono en comparación con la cara a cara resultó en un menor desgaste y una mejora casi equivalente en la depresión en el postratamiento.
Los resultados no son generalizables por haberse tratado sólo un trastorno, además por tratarse

de participantes educados.

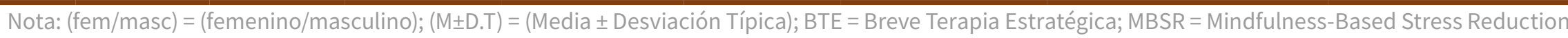
Tabla 3 Intervenciones vía internet

\begin{tabular}{|c|c|c|c|c|c|c|c|c|}
\hline Estudio & $\begin{array}{c}\mathrm{N} \\
\text { (fem/masc) }\end{array}$ & $\begin{array}{c}\text { Edad } \\
\text { M (D.T) }\end{array}$ & Diagnóstico & Diseño & País & Objetivo & Resultados & Limitaciones \\
\hline
\end{tabular}


Eficacia de la Telepsicología en intervenciones del área clínica y de la salud

Una revisión sistemática de la literatura

\begin{tabular}{|c|c|c|c|c|c|c|c|c|}
\hline $\begin{array}{l}\text { Botella et al. } \\
\quad(2010)\end{array}$ & $\begin{array}{c}\text { Grupo } \\
\text { Talk to Me } \\
\text { utoaplicado } \\
(23 / 7) \\
\text { Grupo } \\
\text { Talk to Me } \\
\text { asistido } \\
(18 / 4) \\
\text { Grupo } \\
\text { control lista } \\
\text { de espera } \\
\text { (20/5) }\end{array}$ & $\begin{array}{c}25.9(6.61) \\
22.5(3.44) \\
\text { Rango: } \\
\text { 18-48 años }\end{array}$ & $\begin{array}{c}\text { Trastorno de } \\
\text { ansiedad social }\end{array}$ & $\begin{array}{c}\text { Ensayo } \\
\text { controlado } \\
\text { aleatorizado: } \\
\text { Talk to Me } \\
\text { autoaplicado } \\
\text { vs. Asistido } \\
\text { vs. Lista de } \\
\text { espera }\end{array}$ & España & $\begin{array}{l}\text { Evaluar la eficacia } \\
\text { de "Talk to Me", } \\
\text { un programa de } \\
\text { telepsicología } \\
\text { basado en } \\
\text { internet para el } \\
\text { tratamiento de la } \\
\text { fobia social. }\end{array}$ & $\begin{array}{l}\text { Ambas condiciones } \\
\text { de tratamiento } \\
\text { fueron igualmente } \\
\text { eficaces. Talk to } \\
\text { Me y el mismo } \\
\text { tratamiento aplicado } \\
\text { por un terapeuta } \\
\text { fueron más eficaces } \\
\text { que la condición de } \\
\text { lista de espera. }\end{array}$ & $\begin{array}{l}\text { Desconocimiento de } \\
\text { qué componentes } \\
\text { fueron más eficaces } \\
\text { dado que todos los } \\
\text { participantes recibieron } \\
\text { el mismo programa } \\
\text { (Talk to Me). }\end{array}$ \\
\hline
\end{tabular}

Tabla 3 (continuación)

\begin{tabular}{|c|c|c|c|c|c|c|c|c|}
\hline Estudio & $\begin{array}{c}\mathrm{N} \\
\text { (fem/masc) }\end{array}$ & $\begin{array}{c}\text { Edad } \\
\text { M (D.T) }\end{array}$ & Diagnóstico & Diseño & País & Objetivo & Resultados & Limitaciones \\
\hline $\begin{array}{l}\text { Gershkovich, } \\
\text { Herbert, } \\
\text { Forman \& } \\
\text { Glassman } \\
\text { (2016) }\end{array}$ & $\begin{array}{c}13 \\
(9 / 4)\end{array}$ & $\begin{array}{l}33.2(10.4) \\
\text { Rango: 18-65 } \\
\text { años }\end{array}$ & $\begin{array}{l}\text { Trastorno de } \\
\text { ansiedad social }\end{array}$ & $\begin{array}{l}\text { Pre-Post } \\
\text { tratamiento }\end{array}$ & USA & $\begin{array}{c}\text { Evaluar la } \\
\text { eficacia de una } \\
\text { intervención de } \\
\text { autoayuda basada } \\
\text { en Internet para } \\
\text { el Trastorno de } \\
\text { ansiedad social con } \\
\text { apoyo terapéutico. }\end{array}$ & $\begin{array}{l}\text { Se evidenció una } \\
\text { reducción significativa } \\
\text { en la sintomatología } \\
\text { del trastorno además } \\
\text { de revelar mejorías } \\
\text { en el funcionamiento } \\
\text { psicosocial. Esto } \\
\text { manteniéndose } \\
\text { después de } 3 \text { meses. }\end{array}$ & $\begin{array}{c}\text { Tamaño } \\
\text { reducido de la } \\
\text { muestra. Esto } \\
\text { impidió evaluar } \\
\text { adecuadamente } \\
\text { la hipótesis sobre } \\
\text { los mecanismos } \\
\text { mediación y } \\
\text { moderación. }\end{array}$ \\
\hline $\begin{array}{l}\text { Yuen et al. } \\
\text { (2013b) }\end{array}$ & $\begin{array}{c}14 \\
(8 / 6)\end{array}$ & $\begin{array}{l}36.1 \text { (11.9) } \\
\text { Rango: 21-64 } \\
\text { años }\end{array}$ & $\begin{array}{l}\text { Trastorno de } \\
\text { ansiedad social }\end{array}$ & $\begin{array}{l}\text { Pre-Post } \\
\text { tratamiento }\end{array}$ & USA & $\begin{array}{c}\text { Evaluar la } \\
\text { viabilidad, } \\
\text { aceptabilidad y } \\
\text { eficacia de una } \\
\text { TC basada en la } \\
\text { aceptación de } \\
\text { Second Life para } \\
\text { tratar a adultos con } \\
\text { este trastorno. }\end{array}$ & $\begin{array}{l}\text { Los análisis mostraron } \\
\text { un Pre-tratamiento } \\
\text { significativo a las } \\
\text { mejoras de seguimiento } \\
\text { en los síntomas no sólo } \\
\text { de ansiedad social, sino } \\
\text { depresión. Por lo que se } \\
\text { demostró una TC eficaz. }\end{array}$ & $\begin{array}{c}\text { Varios pacientes } \\
\text { informaron sentir } \\
\text { incomodidad } \\
\text { con revelar } \\
\text { información } \\
\text { personal a un } \\
\text { terapeuta cuya } \\
\text { cara no pudieron } \\
\text { ver. }\end{array}$ \\
\hline
\end{tabular}

Tabla 3 (continuación)

\begin{tabular}{|c|c|c|c|c|c|c|c|c|}
\hline Estudio & $\begin{array}{c}\mathrm{N} \\
\text { (fem/masc) }\end{array}$ & $\begin{array}{c}\text { Edad } \\
\text { M (D.T) }\end{array}$ & Diagnóstico & Diseño & País & Objetivo & Resultados & Limitaciones \\
\hline
\end{tabular}

89

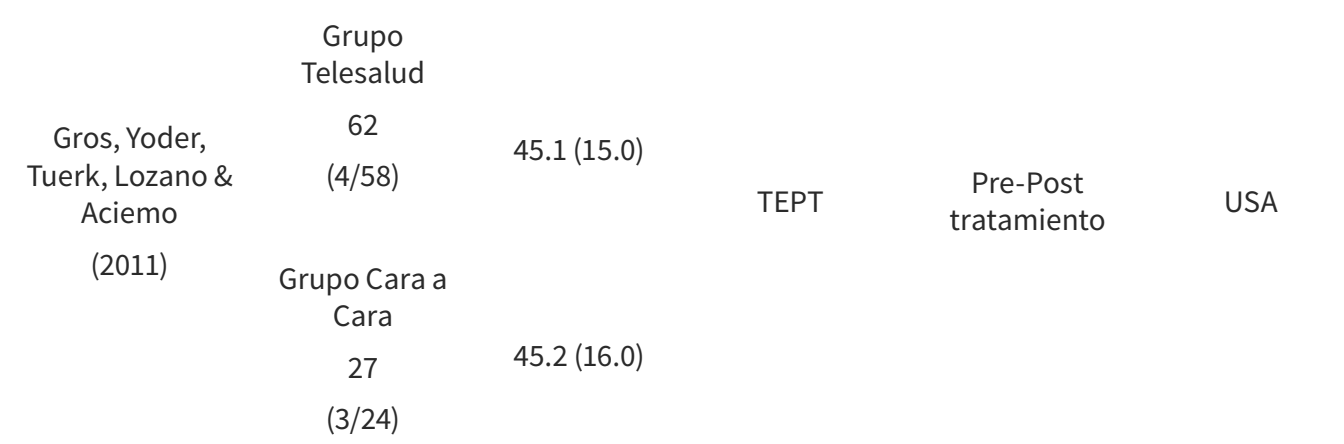

Evaluar la efectividad de la terapia de exposición a veteranos con TEPT a través de telesalud vía internet vs. Cara a cara.
La terapia de exposición por telesalud vía internet La falta de aleatorización demostró eficacia para reducir los síntomas de TEPT además de ansiedad, depresión y estrés. Sin embargo, fue menos eficaz que la terapia cara a cara. entre los dos tipos de intervención pudo resultar en diferencias grupales que afectarían los resultados de los dos tipos de tratamiento.

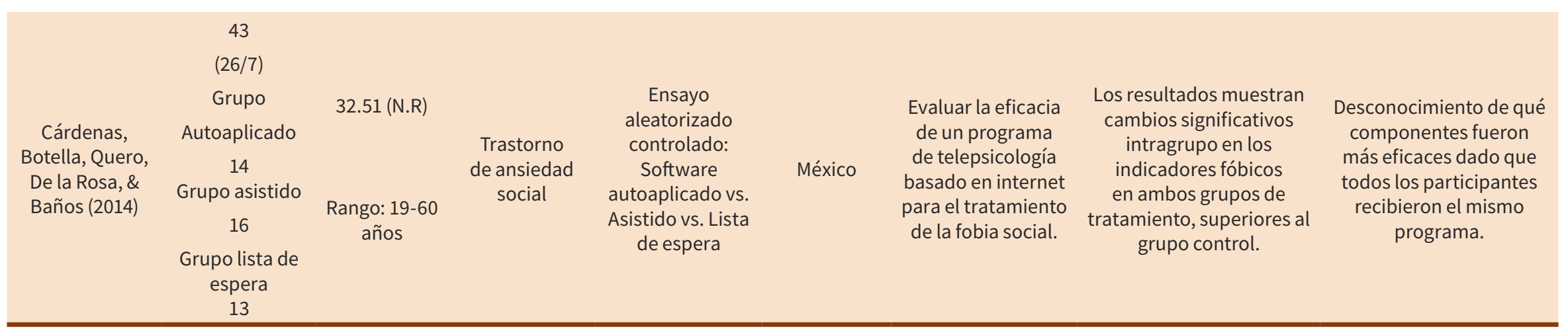


Relacionado al tamaño de la muestra de los estudios, en total 6 artículos usaron intervenciones mediante VC donde el mínimo de sujetos evaluados fue $(n=1)$ y el máximo $(n=202)$. Aquellos que mostraron la intervención vía telefónica evaluaron un total de $(n=391)$ sujetos, y en las investigaciones que usaron la intervención vía internet se evaluaron un mínimo de ( $n=13$ ) sujetos y un máximo de ( $n=658)$. En todas las modalidades de intervención predominaron las participantes femeninas. La edad mínima fue 18 años y la edad máxima fue 92 años.

En las intervenciones de VC, todos los países donde se realizaron los estudios son considerados de renta alta (España, Canadá, Australia y Estados Unidos) y aquellos donde el diseño se caracterizó por ser un ensayo controlado aleatorizado (Germain et al., 2010; Munro-Cullum et al., 2014; Stubbings et al., 2013), los resultados indicaron que la telepsicología fue tan eficaz como en la modalidad cara a cara, no sólo para hacer evaluación sino para efectuar tratamientos neuropsicológicos, considerando ambas modalidades como válidas y confiables con resultados significativos.

Aquellos estudios donde se realizó un pre-post tratamiento (Escudero et al., 2017; Goetter et al., 2014; Yuen et al., 2013a), la telepsicología mediante VC produjo reducciones clínicamente significativas de las sintomatologías tratadas. Y aquellas investigaciones donde se realizó seguimiento a los participantes, estos ya no cumplían con los criterios diagnósticos que una vez tenían, y los análisis revelaban así mismo un pretratamiento significativo en las mejoras de aquellos seguimientos, todo esto comparable con la terapia cognitivo conductual tradicional.

En las intervenciones vía telefónica se emplearon diseños caracterizados por ser pre-post tratamiento (Ben-Seev et al., 2014) y ensayo controlado aleatorizado (Himelhoch et al., 2013; Mohr et al., 2012). En el primer diseño el diagnóstico a tratar fue la esquizofrenia, y los resultados mostraron reducciones significativas en los síntomas psicóticos y en la psicopatología general después de un mes de uso, mostrando así la eficacia de esta modalidad de intervención. Por otra parte, aquellos autores que emplearon el segundo diseño mencionado anteriormente, evaluaron el trastorno de depresión mayor, llegando a la conclusión que tanto por teléfono como cara a cara se reducen significativamente los síntomas depresivos, consiguiendo igualmente un menor desgaste en los participantes y resultados eficaces comparado con la modalidad de intervención tradicional.

En la telepsicología vía internet, todos los estudios se realizaron en países considerados de renta alta, excepto México, considerado como país de renta media alta. El trastorno que más recibió intervención en este tipo de modalidad fue el trastorno de ansiedad social, y mediante ensayos controlados aleatorizados (Botella et al., 2010; Cárdenas et al., 2014) donde los grupos estuvieron divididos entre aquellos que recibían atención mediante un software autoaplicado vs. Asistido por un terapeuta vs. Lista de espera, los resultados concuerdan en que ambas condiciones de tratamiento (sincrónica/asincrónica) fueron igualmente eficaces aunque las puntuaciones hayan sido inferiores en el grupo control.

La fobia social también fue intervenida vía internet en estudios con diseños pre-post tratamiento (Gershkovich et al., 2016; Yuen et al., 2013b), donde se evidenció en ambas investigaciones no sólo una reducción significativa de la sintomatología sino una mejoría tanto en el funcionamiento psicosocial como en los síntomas depresivos, demostrando una viabilidad, aceptabilidad y eficacia alta de este tipo de intervención.

Todos los estudios en la telepsicología vía internet mostraron que este tipo de modalidad es tan eficaz como en la intervención cara a cara, sin embargo, en una investigación con un diseño metodológico pre-post tratamiento (Gros et al., 2011), donde se evaluaba la efectividad de la terapia de exposición de veteranos con TEPT, se encontró aunque la exposición por telesalud demostró eficacia para reducir la sintomatología, resultó siendo menos eficaz que la terapia tradicional.

Como limitaciones, las que más se registraron en las intervenciones donde se usó la VC fue el pequeño tamaño de la muestra (Goetter et al., 2014; Stubbings et al., 2013) y problemas técnicos durante las sesiones, o escenarios online limitados que dificultaban los ejercicios de exposición (Germain et al., 2010; Munro-Cullum et al., 2014; Yuen et al., 2013a). En el caso de la intervención vía telefónica la ausencia de grupo control y la no generalización de los resultados fueron algunas de las limitaciones registradas (Be-Seev et al., 2014; Mohr et al., 2012). Por último, algunos resultados de las intervenciones mediante internet pudieron verse afectados por la incomodad de los participantes al revelar información personal a alguien que no podían ver (Yuen et al., 2013b), como también el no poder ser generalizados si la intensidad de la sintomatología es mayor (Cheng et al., 2018).

\section{Discusión}

El objetivo de esta revisión fue evaluar la efectividad de la telepsicología mediante VC, teléfono e internet en el área clínica y de la salud para el tratamiento de trastornos mentales en los últimos diez años. La literatura revisada sugiere que existe una base de evidencia con rápido crecimiento en esta área los últimos 10 años, y en términos generales todos los estudios de la muestra confirmaron no sólo la alta efectividad de estas modalidades de intervención, sino la viabilidad y confiabilidad de estas en el área clínica y de la salud.

Dentro de las tres modalidades de telepsicología revisadas, aquella con más escasez de estudios fue la empleada de forma telefónica. Esto conlleva a cuestionar si puede haber un sesgo de publicación en la literatura para esta modalidad de tratamiento en el que los resultados nulos no son publicados o que efectivamente se han realizado muy pocos tratamientos por teléfono. Sin embargo, se percibe que aunque este tipo de intervención cuenta con menos estudios, los resultados muestran tanta efectividad como aquellos donde se utilizó la VC y el internet.

De igual forma, en términos generales se percibe que los estudios muestran una alta satisfacción de los participantes con las intervenciones psicoterapéuticas en la modalidad virtual comparado con la modalidad cara a cara, sin embargo, dichas investigaciones han contado a lo largo de los años con un tamaño reducido de la muestra, lo que puede limitar las conclusiones y conllevar a cuestionamientos sobre si aquellos estudios donde no se evidenció gran eficacia de la telepsicología, tendrían relación con el grado de satisfacción o con el resultado clínico en el proceso de tratamiento independientemente si la terapia fue por VC, vía telefónica o basada en internet.

Las intervenciones en los estudios que conformaron la muestra final, por otro lado, se caracterizaron por ser la mayoría de forma sincrónica $(n=11)$, y $n=4$ por ser de forma asincrónica. Aquellas investigaciones donde se intervino mediante VC $(n=6)$ se realizaron todas de manera sincrónica; 2 de los 3 estudios encontrados en la modalidad telefónica (Himelhoch et al., 2013; Mohr et al., 2012) así como 3 estudios donde se intervino mediante internet (Gershkovich et al., 2016; Yuen et al., 2013b; Gros et al., 2016) también se realizaron de forma simultánea entre terapeuta y paciente. Los resultados mostraron no sólo la efectividad de la telepsicología sincrónica, sino una gran aceptabilidad por parte de los participantes, que se compara con el mismo grado de satisfacción en intervenciones cara a cara. 


\section{Eficacia de la Telepsicología en intervenciones del área clínica y de la salud}

Una revisión sistemática de la literatura

Aquellos estudios asincrónicos (Ben-Seev et al., 2014; Cheng et al., 2018; Botella et al., 2010; Cárdenas et al., 2014), a pesar de no contar con una relación simultánea con el terapeuta, los resultados mostraron eficacia con resultados clínicamente significativos en tratamientos autoaplicados así como reducciones significativas en la sintomatología de trastornos psicológicos, mostrando igualmente una alta efectividad de la telepsicología asincrónica comparada con intervenciones tradicionales.

Se observa, además, que la tecnología empleada en telepsicología es relativamente nueva, particularmente en el caso de las intervenciones administradas con VC e internet. Esto se refleja por una mayor cantidad de estudios con esta modalidad de intervención en la última década, razón por la cual la presente investigación se enfocó en analizar estos estudios durante este rango tiempo y sugiere, además, la importancia de seguir estudiando estas modalidades de intervención puesto que es probable que la evidencia para las intervenciones de VC e internet continúe creciendo a un ritmo mayor en los próximos años.

Esta revisión, a su vez, ayuda a reconocer el potencial de la telepsicología en superar las barreras comunes para el acceso al tratamiento, demostrando gran eficacia y equivalencia a los tratamientos tradicionales. Además, parece ser un método de intervención prometedor que aumenta el alcance de las intervenciones psicológicas basadas en la evidencia, permitiendo el acceso a psicoterapias eficaces a comunidades rurales y remotas que lo necesiten.

Es importante considerar algunas limitaciones de la presente revisión, una de estas fue que sólo se tuvieron en cuenta tres modalidades de intervención en telepsicología (VC, vía internet y vía telefónica), así mismo, sólo se dio un enfoque a aquellos tratamientos en el área clínica y de la salud, sería importante para futuras revisiones tener en cuenta otros campos de la psicología que ayudarían a ampliar y reconocer la efectividad de las ciber intervenciones en otros contextos como los escolares o sociales, así como en otros tipos de muestras, es decir, no sólo personas con trastornos mentales, una propuesta sería sujetos considerados minorías raciales o étnicas.

\section{Referencias}

Acero, P., Cabas, K., Caycedo, C., Figueroa, P., Patrick, G., \& Rudas, M. M. (2020). Telepsicología: sugerencias para la formación y el desempeño professional responsable. Recuperado de: https://www.colpsic.org. co/aym_image/files/GUIA\%20TELEPSICOLOGIA\%20COLOMBIA\%20 PRIMER\%20DOCUMENTO\%20final.pdf

American Psychological Association. (2013). Guidelines for the practice of telepsychology. Recuperado de: https://www.apa.org/practice/ guidelines/telepsychology

Backhaus, A., Agha, Z., Maglione, M. L., Repp, A., Ross, B., Zuest, D.,... Thorp, S. R. (2012). Videoconferencing psychotherapy: A systematic review. Psychological services, 9(2), 111-131. Recuperado de: https:// pubmed.ncbi.nlm.nih.gov/22662727/

Been-Zeev, D., Brenner, C. J., Begale, M., Duffecy, J., Mohr, D. C., \& Mueser, K. T. (2014). Feasibility, Acceptability, and Preliminary Efficacy of a Smartphone Intervention for Schizophrenia. Squizophrenia Bulletin, 40(6), 1244-1253. Recuperado de: https://academic.oup.com/ schizophreniabulletin/article/40/6/1244/1854428

Botella, C., Gallego, M. J., García-Palacios, A., Guillen, V., Baños, R. M., Quero, S., \& Alcañiz, M. (2010). An Internet-Based Self-Help Treatment for Fear of Public Speaking: A Controlled Trial. Cyberpsychology, Behavior and Social Networking, 13(4), 407-421. Recuperado de: https://www.liebertpub.com/doi/10.1089/cyber.2009.0224
Cárdenas, G., Botella, C., Quero, S., De la Rosa, A., \& Baños, R. (2014). Programa de telepsicología para el tratamiento de la fobia a hablar en público en población mexicana. Psicología Iberoamericana, 22(1), 45-54. Recuperado de: https://www.redalyc.org/ pdf/1339/133944229006.pdf

Cheng, P., Luik, A. I., Fellman-Couture, C., Peterson, E., Joseph, C. L. M., Tallent, G.,... Drake, C. L. (2018). Efficacy of digital CBT for insomnia to reduce depression across demographic groups: a randomized trial. Psychological Medicine, 49(3), 491-500. Recuperado de: https:// pubmed.ncbi.nlm.nih.gov/29792241/

Clinton, B. K., Silverman, B. C., \& Brendel, D. H. (2010). Patient-targeted googling: The ethics of searching online for patient information. Harvard Review of Psychiatry, 18(2), 103-112. Recuperado de: https://pubmed.ncbi.nlm.nih.gov/20235775/

DANE. (2019). ¿Dónde estamos? Recuperado de: https://www.dane.gov.co/ index.php/estadisticas-por-tema/demografia-y-poblacion/censonacional-de-poblacion-y-vivenda-2018/donde-estamos

DiLillo, D., \& Gale, E. B. (2011). To google or not to google: Graduate students' use of the Internet to access personal information about clients. Training and Education in Professional Psychology, 5(3), 160-166. Recuperado de: https://psycnet.apa.org/ record/2011-17345-006

Downs, S. H., \& Black, N. (1998). The feasibility of creating a checklist for the assessment of the methodological quality both of randomized and non-randomised studies of health care interventions. 1(52), 377384. Recuperado de: https://www.ncbi.nlm.nih.gov/pmc/articles/ PMC1756728/pdf/v052p00377.pdf

Drum, K. B., \& Littleton, H. L. (2014). Therapeutic Boundaries in Telepsychology: Unique Issues and Best Practice Recommendations. Professional Psychology: Research and Practice, 45(5), 309-315. Recuperado de: https://psycnet.apa.org/record/2014-09407-001

Escudero, S., Navarro, R., Reguera, B., Gesteira, C., Morán, N., García-Vera, M. P., \& Sanz, J. (2018). Tratamiento Psicológico por Videoconferencia de una Víctima de Terrorismo con Trastorno por Estrés Postraumático y Otros Trastornos Emocionales Comórbidos 30 Años después del Atentado. Clínica y Salud, 29(1), 21-26. Recuperado de: https://journals.copmadrid.org/clysa/art/clysa2018a4

Ferreras-Fernández, T., Martín-Rodero, H., García-Peñalvo, F. J., \& MerloVega, J. A. (2016). The Systematic Review of Literature in LIS: An approach. In F. J. García-Peñalvo (Ed.), Proceedings of the Fourth International Conference on Technological Ecosystems for Enhancing Multiculturality (TEEM'16) (pp. 291-298). New York, USA: ACM. Recuperado de: https://dl.acm.org/doi/10.1145/3012430.3012531

Frueh, B. C. (2015). Solving mental healthcare access problems in the twenty-first century. Australian Psychologist, 50(4), 304306. Recuperado de: https://aps.onlinelibrary.wiley.com/doi/ abs/10.1111/ap.12140

Germain, V., Marchand, A., Bouchard, S., Guay, S., \& Drouin, M. S. (2010). Assessment of the Therapeutic Alliance in Face-to-Face or Videoconference Treatment for Posttraumatic Stress Disorder. Cyberpsychology, Behavior, and Social Networking, 13(1), 29-35. Recuperado de: https://pubmed.ncbi.nlm.nih.gov/20528290/

Gershkovich, M., Herbert, J. D., Forman, E. M., \& Glassman, L. (2016). Guided Internet-Based Self-Help Intervention for Social Anxiety Disorder With Videoconferenced Therapist Support. Cognitive and Behavioral Practice, 23(2), 239-255. Recuperado de: https://psycnet.apa.org/ record/2015-47071-001

Goetter, E. M., Herbert, J. D., Forman, E. M., Yuen, E. K., \& Thomas, J. G. (2014). An open trial of videoconference-mediated exposure and ritual prevention for obsessive-compulsive disorder. Journal of Anxiety Disorders, 28(5), 460-462. Recuperado de: https://www. sciencedirect.com/science/article/abs/pii/S0887618514000668

Gros, D. F., Yoder, M., Tuerk, P. W., Lozano, B. E., \& Acierno, R. (2011). Exposure therapy for PTSD delivered to veterans via telehealth: predictors of treatment completion and outcome and comparison to treatment delivered in person. Behavior therapy, 42(2), 276-283. Recuperado de: https://pubmed.ncbi.nlm.nih.gov/21496512/ 
Handley, T. E., Kay-Lambkin, F. J., Inder, K. J., Lewin, T. J., Attia, J. R., Fuller, J.,... Kelly, B. J. (2014). Self-reported contacts for mental health problems by rural residents: predicted service needs, facilitators and barriers. BMC Psychiatry, 14(1), 249. Recuperado de: https:// bmcpsychiatry.biomedcentral.com/articles/10.1186/s12888-0140249-0

Hilty, D. M., Shoemaker, E. Z., Myers, K., Snowdy, C. E., Yellowlees, P. M., \& Yager, J. (2016). Need for and Steps Toward a Clinical Guideline for the Telemental Healthcare of Children and Adolescents. Journal of child and adolescent psychopharmacology, 26(3), 283-295. Recuperado de: https://www.liebertpub.com/doi/10.1089/cap.2015.0129

Himelhoch, S., Medoff, D., Maxfield, J., Dihmes, S., Dixon, L., Robinson, C.,.. Mohr, D. C. (2013). Telephone Based Cognitive Behavioral Therapy Targeting Major Depression Among Urban Dwelling, Low Income People Living with HIV/AIDS: Results of a Randomized Controlled Trial. Aids and Behavior, 17(8), 2756-2764. Recuperado de: https:// link.springer.com/article/10.1007/s10461-013-0465-5

Jenkins-Guarnieri, M. A., Pruitt, L. D., Luxton, D. D., \& Johnson, K. (2015). Patient Perceptions of Telemental Health: Systematic Review of Direct Comparisons to In-Person Psychotherapeutic Treatments. Telemedicine journal and e-health: the official journal of the American Telemedicine Association, 21(8), 652-660. Recuperado de: https://pubmed.ncbi.nlm.nih.gov/25885491/

Manterola, C., Astudillo, P., Arias, E., \& Claros, N. (2013). Revisiones sistemáticas de la literatura. Qué se debe saber acerca de ellas. Cirugía Española, 91(3), 149-155. Recuperado de: https://www. sciencedirect.com/science/article/abs/pii/S0009739X11003307

Meadows, G. N., Enticott, J. C., Inder, B., Russell, G. M., \& Gurr, R. (2015). Better access to mental health care and the failure of the Medicare principle of universality. Medical Journal of Australia, 202(4), 190-194. Recuperado de: https://onlinelibrary.wiley.com/doi/abs/10.5694/ mja14.00330

Mehrotra, A., Jena, A. B., Busch, A. B., Souza, J., Uscher-Pines, L., \& Landon, B. E. (2016). Utilization of telemedicine among rural Medicare beneficiaries. Jama, 315(18), 2015-2016. Recuperado de: https:// jamanetwork.com/journals/jama/fullarticle/2520619

Moher, D., Liberati, A., Tetzlaff, J., Altman, D. G., \& The Prisma Group (2009). Preferred Reporting Items for Systematic Reviews and Meta-Analyses: The PRISMA Statement. PLoS Medicine, 6(7), 1-6. Recuperado de: https://journals.plos.org/plosmedicine/article?id=10.1371/journal. pmed.1000097

Mohr, D. C., Ho, J., Duffecy, J., Reifler, D., Sokol, L., Burns, M. N.,... Siddique, J. (2012). Effect of Telephone-Administered vs Face-To-Face Cognitive Behavioral Therapy on Adherence to Therapy and Depression Outcomes Among Primary Care Patients: A Randomized Trial. JAMA, 307(21), 2278-2285. Recuperado de: https://jamanetwork.com/ journals/jama/fullarticle/1172045

Munro-Cullum, C., Hynan, L. S., Grosch, M., Parikh, M., \& Weiner, M. F. (2014). Teleneuropsychology: Evidence for Video Teleconference-Based Neuropsychological Assessment. Journal of the International Neuropsychological Society, 20(10), 1028-1033. Recuperado de: https://www.cambridge.org/core/journals/journal-ofthe-international-neuropsychological-society/article/abs/ teleneuropsychology-evidence-for-video-teleconferencebasedneuropsychological-assessment/0AD8A5F437443774924386FEF69 $1 \mathrm{~A} 171$

Nelson, E. L., Bui, T. N., \& Velásquez, S. E. (2011). Telepsychology: Research and practice overview. Child and Adolescent Psychiatric Clinics of North America, 20(1), 67-79. Recuperado de: https://pubmed.ncbi. nlm.nih.gov/21092913/

Osenbach, J. E., O’Brien, K. M., Mishkind, M., \& Smolenski, D. J. (2013). Synchronous telehealth technologies in psychotherapy for depression: A meta-analysis. Depression and Anxiety, 30(11), 10581067. Recuperado de: https://onlinelibrary.wiley.com/doi/10.1002/ da. 22165
Pati, D., \& Lorusso, L. N. (2017). How to write a Systematic Review of the Literature. Heatlh Environments Research \& Design Journal, 11(1), 1-6. Recuperado de: https://journals.sagepub.com/ doi/10.1177/1937586717747384

Reilly-Spong, M., Reibel, D., Pearson, T., Koppa, P., \& Gross, C. R. (2015). Telephone-adapted mindfulness-based stress reduction (tMBSR) for patients awaiting kidney transplantation: Trial design, rationale and feasibility. Contemporary Clinical Trials, 42(1), 169-184. Recuperado de: https://pubmed.ncbi.nlm.nih.gov/25847578/

Riding-Malon, R., \& Werth Jr, J. L. (2014). Psychological practice in rural settings: At the cutting edge. Professional Psychology: Research and Practice, 45(2), 85. Recuperado de: https://psycnet.apa.org/ record/2014-13439-001

Soto-Pérez, F., \& Franco-Martín, M. (2018). Atención psicológica y Tecnologías: oportunidades y conflictos. Revista Iberoamericana de Psicología, 11(3), 109-120. Recuperado de: https:// reviberopsicologia.ibero.edu.co/article/view/rip.11310/pdf

Stubbings, D. R., Rees, C. S., Roberts, L. D., \& Kane, R. T. (2013). Comparing In-Person to Videoconference-Based Cognitive Behavioral Therapy for Mood and Anxiety Disorder: Randomized Controlled Trial. Journal of Medical Internet Research, 15(11), 1-17. Recuperado de: https:// www.jmir.org/2013/11/e258/

Sucala, M., Schnur, J. B., Constantino, M. J., Miller, S. J., Brackman, E. H., \& Montgomery, G. H. (2012). The therapeutic relationship in e-therapy for mental health: a systematic review. Journal of medical Internet research, 14(4), e: 110. Recuperado de: https://www.jmir.org/2012/4/ e110/

Tudor-Tulbure, B. (2011). The efficacy of Internet-supported intervention for social anxiety disorder: A brief meta-analytic review. Procedia Social and Behavioral Sciences, 30(1), 552-557. Recuperado de: https:// www.sciencedirect.com/science/article/pii/S1877042811019331

Varker, T., Brand, R. M., Ward, J., Terhaag, S., \& Phelps, A. (2018). Efficacy of synchronous telepsychology interventions for people with anxiety, depression, posttraumatic stress disorder and adjustment disorder: A rapid evidence assessment. Psychological Services, 16(4), 621-635. Recuperado de: https://www.researchgate. net/publication/325437378_Efficacy_of_Synchronous Telepsychology_Interventions_for_People_With_Anxiety Depression_Posttraumatic_Stress_Disorder_and_Adjustment Disorder_A_Rapid_Evidence_Assessment

Wilson, L. S., \& Maeder, A. J. (2015). Recent directions in telemedicine: Review of trends in research and practice. Healthcare informatics research, 21(4), 213-222. Recuperado de: https://pubmed.ncbi.nlm. nih.gov/26618026/

Yuen, E. K., Herbert, J. D., Forman, E. M., Goetter, E. M., Juarascio, A. S., Rabin, S.,... Bouchard, S. (2013a). Acceptance based behavior therapy for social anxiety disorder through videoconferencing. Journal of Anxiety Disorders, 27(4), 389-397. Recuperado de: https://www. sciencedirect.com/science/article/abs/pii/S0887618513000388

Yuen, E. K., Herbert, J. D., Forman, E. M., Goetter, E. M., Comer, R., \& Bradley, J.C. (2013b). Treatment of Social Anxiety Disorder Using Online Virtual Environments in Second Life. Behavior Therapy, 44(1), 51-61. Recuperado de: https://pubmed.ncbi.nlm.nih.gov/23312426/ 